\title{
Frustration-induced dodecamer ordering in the double-exchange spin ice model on the kagomé lattice
}

\author{
Yoshihiro Shimomura*, Shin Miyahara and Nobuo Furukawa \\ Department of Physics and Mathematics, Aoyama Gakuin University, Sagamihara 229-8558, Japan
}

(Received November 30, 2018)

\begin{abstract}
We investigate a detail of a dodecamer cluster ordering in a double-exchange spin ice model on a kagomé lattice. In frustrated systems, ordinary spin orderings are suppressed and macroscopic degeneracy remains down to low temperatures. In some frustrated systems, the degeneracy is lifted due to residual interactions and cluster orderings are stabilized. In the present model, the spin ice state is first formed at intermediate temperatures, and further entropies are released at lower temperatures as the dodecamer phase emerges. Since the spin symmetry is not broken in the dodecamer phase, there still exists macroscopic degeneracy. At further low temperatures, a possible spin ordering due to inter-dodecamer interactions is proposed. We discuss that such a multiple-site clustering larger than a bond-pair might be generic to frustrated systems where macroscopic degeneracy is lifted by residual interactions.

KEYWORDS: dodecamer, cluster order, double-exchange model, kagomé lattice, frustration, metal, spin ice, Monte Carlo simulation
\end{abstract}

\section{Introduction}

Intensive studies have been devoted to frustrated systems because of their unexpected behaviors. In spin systems, a frustration arises when all interaction energies between localized spins can not be minimized simultaneously in the classical picture. It is known that the frustration suppresses the long-range order and often induces the novel ground state. The macroscopically degenerate ground state accompanied with a residual entropy is one of the well-known states in frustrated Ising spin systems. Such a state is seen in the antiferromagnetic (AF) Ising model on a triangular, ${ }^{1}$ a kagomé ${ }^{2,3}$ and a pyrochlore ${ }^{4}$ lattices. On the other hand, a peculiar cluster ordered state can be induced by the frustration. In the Majundar-Ghosh model $^{5}$ and the Shastry-Sutherland model ${ }^{6}$ which are frustrated quantum spin systems, the dimer-singlet states with a spin gap are realized as the unique ground states. The entropies in the systems are released by the dimer-singlet formations for all localized spins, which can be viewed as a cluster ordering since the systems are tiled by the dimer-singlets. Such dimer-singlet ground states are driven by geometrical frustrations and quantum effects of the interactions. The cluster ordering can be the way to stabilize the states in the frustrated systems.

*E-mail address: shimomura@phys.aoyama.ac.jp 

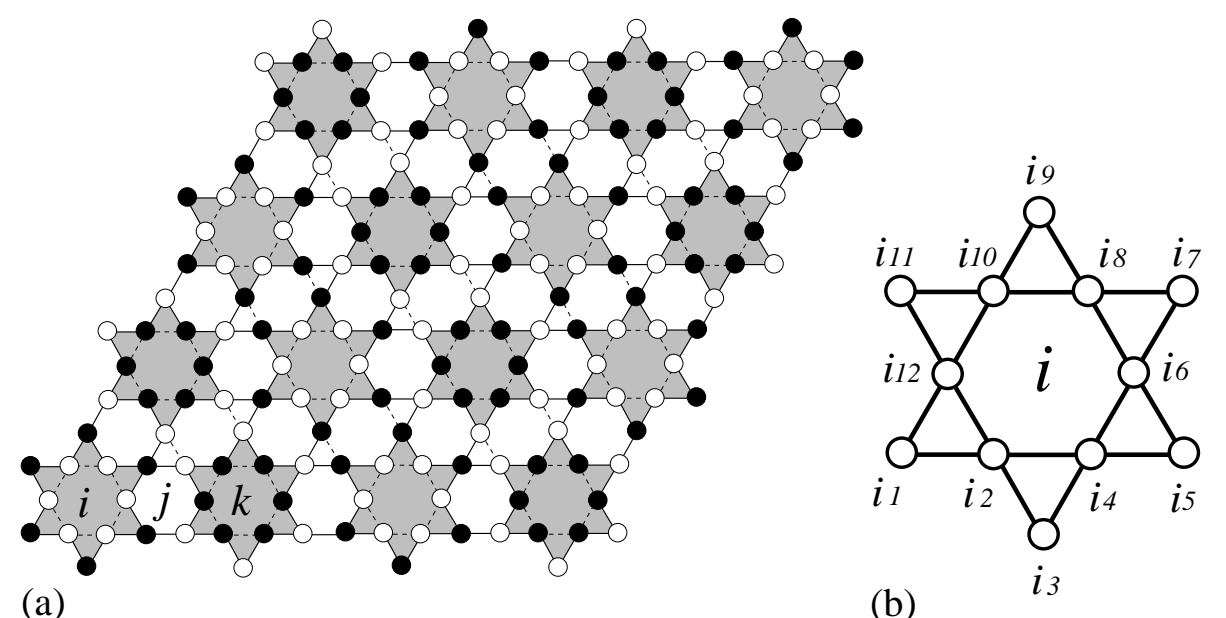

Fig. 1. (a) Dodecamer phase. Open and closed circles represent out- and in-spins for the up-triangles, respectively. The dodecamer is represented by the shadowed spin cluster. Each dodecamer is labeled $i, j, k$ and others. (b) Twelve sites which construct the $i$-th dodecamer.

Actually, such a cluster order is seen even in realistic materials. For example, the Shastry-Sutherland model is realized in the two-dimensional spin- $1 / 2$ orthogonal dimer system $\mathrm{SrCu}_{2}\left(\mathrm{BO}_{3}\right)_{2}$, where $\mathrm{Cu}$ sites are tiled by the dimer-singlet. This material is novel in terms of the existence of the exact dimer-singlet ground state with the finite spin gap in the two-dimensional system. ${ }^{7,8}$ Furthermore, as possible other experimental examples of the spin cluster order in frustrated spin systems, hexamers of $\mathrm{Cr}^{3+}$ in a cubic spinel $\mathrm{ZnCr}_{2} \mathrm{O}_{4}{ }^{9}$ and octamers of $\mathrm{Ir}^{3+}$ and $\mathrm{Ir}^{4+}$ in a thiospinel $\mathrm{CuIr}_{2} \mathrm{~S}_{4}{ }^{10}$ have been reported.

In this way, cluster orders in frustrated spin systems are seen in both theoretical and experimental researches, and have attracted much attentions. Even in electron systems, similar cluster ordering induced by the frustration can be realized. As an example of such cluster orders in electron systems, we have reported dodecagonal localized spin cluster, "dodecamer", order in a double-exchange spin ice (DESI) model on the kagomé lattice ${ }^{11}$ [see Fig. 1(a)]. In the DESI model, localized spins have local uniaxial anisotropies for triangles which constitute the kagomé lattice: Localized spins are forced to point in the directions connecting the center of mass and the three vertexes of the triangles, that is, they point either inward (in-spin) or outward (out-spin) for up-triangles. There is an effective ferromagnetic interaction between localized spins due to the double-exchange (DE) mechanism. ${ }^{12}$ The situation that there are the ferromagnetic interactions and the uniaxial anisotropies for localized spins is similar to that in the spin ice systems. ${ }^{13}$ From an analogy with the spin ice systems, it is obvious that the DESI model has the frustration and a spin-ice-like behavior. The dodecamer formation is driven by the frustration and the kinetic energy gain due to the DE mechanism: At sufficiently low temperatures, electrons move along a certain selected path to gain the kinetic energy 
under the frustration. The dodecamer formation is a consequence of the path selection by electrons in order to gain the kinetic energy. In the dodecamer phase, the kagomé lattice can be completely tiled by dodecamers, which leads to the dodecamer order with the transilational symmetry breaking. Note that there are two kinds of dodecamers: One is the dodecamer with in-spins on the tips $i_{2 n-1}(n=1,2, \cdots, 6)$ shown in Fig. 1(b) and the other is out-spins. From the existence of two dodecamers, it is found that the spin symmetry is not broken in the dodecamer phase.

In this paper, we focus on the behaviors of the entropy release in the DESI system. As the temperature is lowered, the thermodynamics in the DESI system is as follows. (1) The spin-ice-like state is stabilized at intermediate temperatures. (2) The dodecamer order appears at sufficiently low temperatures. In the spin-ice-like state, there still remain a large part of degrees of freedom for localized spins, i.e., there is a finite entropy which is almost consistent with the residual entropy of the AF Ising model on the kagomé lattice. ${ }^{3}$ A large part of the entropy is released by the dodecamer ordering. There is a possibility that the entropy still remains even in the dodecamer phase, since each dodecamer is two-fold degenerate.

\section{Double-Exchange Spin Ice Model}

In this section, let us introduce the DESI model on the kagomé lattice, which is the Anderson-Hasegawa model ${ }^{14}$ with local uniaxial anisotropies. The mechanism for the frustration in this system is similar to that in the spin ice systems. ${ }^{13}$ It is expected that similar degeneracy as in the spin ice systems exists in the DESI model.

\subsection{Anderson-Hasegawa model}

The Anderson-Hasegawa model ${ }^{14}$ is a modified DE model with infinitely large Hund's-rule couplings:

$$
\hat{H}=-\sum_{\langle i, j\rangle} t\left(\boldsymbol{S}_{i}, \boldsymbol{S}_{j}\right)\left(c_{i}^{\dagger} c_{j}+h . c .\right)-\mu \sum_{i} c_{i}^{\dagger} c_{i},
$$

where $c_{i}^{\dagger}\left(c_{i}\right)$ is an electron operator which creates (annihilates) an electron with a spin parallel to a localized spin at site $i, \boldsymbol{S}_{i}$, and $\mu$ is the chemical potential in the grand canonical ensemble. Here, $t\left(\boldsymbol{S}_{i}, \boldsymbol{S}_{j}\right)$ is given in the form,

$$
t\left(\boldsymbol{S}_{i}, \boldsymbol{S}_{j}\right)=t \cos \left(\frac{\theta_{i j}}{2}\right)
$$

where $t$ is a transfer integral and $\theta_{i j}$ is a relative angle between the localized spins $\boldsymbol{S}_{i}$ and $\boldsymbol{S}_{j}$. The angle $\theta_{i j}$ is given by

$$
\cos \theta_{i j}=\cos \theta_{i} \cos \theta_{j}+\sin \theta_{i} \sin \theta_{j} \cos \left(\phi_{i}-\phi_{j}\right)
$$

where $\left(\theta_{i}, \phi_{i}\right)$ is an angle of $\boldsymbol{S}_{i}$ in the polar coordinate. Note that $t\left(\boldsymbol{S}_{i}, \boldsymbol{S}_{j}\right)$ has a maximum value $t$ when $\boldsymbol{S}_{i}$ and $\boldsymbol{S}_{j}$ are parallel. Thus, ferromagnetic alignment of the localized spins broadens the bandwidth of the system, which produces a kinetic energy gain. Therefore, there 


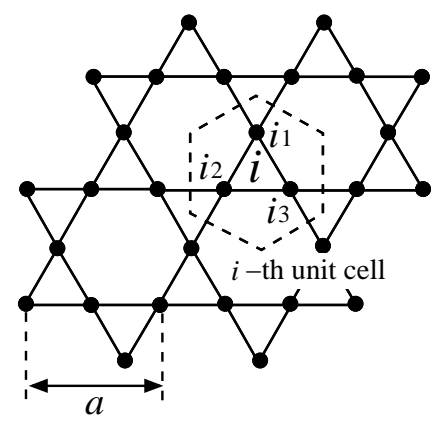

(a) kagome lattice
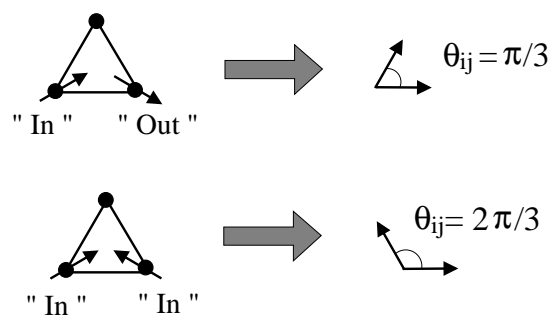

(c) two relative angles

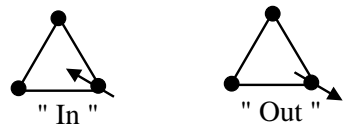

(b) uniaxial anisotropies
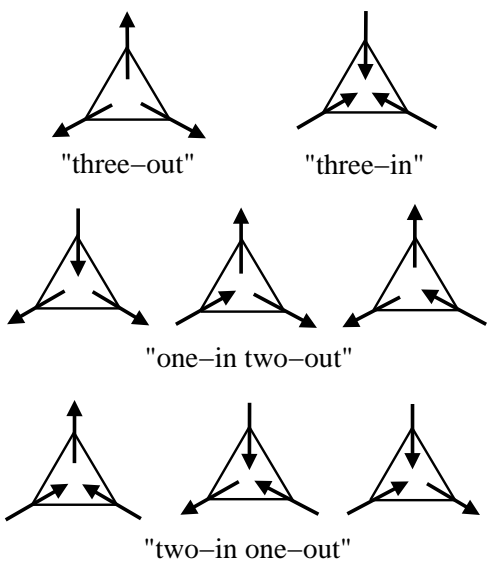

(d) possible eight spin configurations

Fig. 2. (a) Kagomé lattice. The $i$-th unit cell surrounded by the dashed line consists of three sites $i_{1}, i_{2}$ and $i_{3} . a$ is a lattice constant. (b) Uniaxial anisotropies for localized spins. Localized spins have two degrees of freedom, "in" and "out", for a triangle. (c) two relative angles in the DESI system: The relative angle $\theta_{i j}$ between in-spin and out-spin, and between the same spins, are $\frac{\pi}{3}$ and $\frac{2 \pi}{3}$, respectively. (d) Possible eight configurations for an up-triangle in the kagomé lattice.

is an effective ferromagnetic interaction between the nearest-neighbor (n.n.) localized spins. We set the transfer integral $t \equiv 1$, hereafter.

\subsection{Frustrated electron system : double-exchange spin ice model}

We consider the Anderson-Hasegawa model on the kagomé lattice [see Fig. 2(a)], and introduce uniaxial anisotropies for the localized spins as follows: They are forced to be either in-spin or out-spin for up-triangles on the kagomé lattice [see Fig. 2(b)]. Under the conditions, relative angles between the localized spins, $\theta_{i j}$ (Eq.(2)), can take only two values, i.e., $\theta_{i j}^{1} \equiv \pi / 3$ (the angle between an in-spin and an out-spin) and $\theta_{i j}^{2} \equiv 2 \pi / 3$ (the angle between the same spins) [see Fig. 2(c)]: The transfer integral of electrons $t\left(\boldsymbol{S}_{i}, \boldsymbol{S}_{j}\right)$ has $t_{1} \equiv \cos \left(\theta_{i j}^{1} / 2\right)=\sqrt{3} / 2$ and $t_{2} \equiv \cos \left(\theta_{i j}^{2} / 2\right)=1 / 2$. Thus, electrons can easily move through the ferromagnetic bonds with the transfer integral $t_{1}$ due to the kinetic energy gain.

To clarify the frustration in the DESI system, we introduce a pseudospin representation. We define a pseudospin corresponding to $\boldsymbol{S}_{i}$,

$$
\tau_{i}= \begin{cases}+1 & \left(\boldsymbol{S}_{i} \text { is an out-spin }\right) \\ -1 & \left(\boldsymbol{S}_{i} \text { is an in-spin }\right)\end{cases}
$$


In this pseudospin picture, the Hamiltonian (1) is rewritten in the form

$$
\hat{H}=\sum_{\langle i, j\rangle} t\left(\tau_{i}, \tau_{j}\right)\left(c_{i}^{\dagger} c_{j}+\text { h.c. }\right)-\mu \sum_{i} c_{i}^{\dagger} c_{i},
$$

where

$$
t\left(\tau_{i}, \tau_{j}\right)= \begin{cases}-t_{1} & \left(\tau_{i}=-\tau_{j}\right), \\ -t_{2} & \left(\tau_{i}=\tau_{j}\right) .\end{cases}
$$

The effective ferromagnetic interaction between the n.n. localized spins is regarded as the effective AF interaction between the n.n. pseudospins. Thus, there is the n.n. AF interaction on the kagomé lattice. Using the pseudospin picture, it is easily understood that the system has the frustration.

\subsection{Spin ice state in the double-exchange spin ice model}

In the DESI system, each triangle has four types of spin configurations, i.e., "three-in", "three-out", "two-in one-out" and "one-in two-out" as shown in Fig. 2(d). These correspond to "three-down", "three-up", "two-down one-up" and "one-down two-up", respectively, in the pseudospin picture. Naturally, as the temperature is lowered, three-in and three-out configurations are unfavored by the ferromagnetic interaction due to the DE mechanism. In that case, the majority of localized spin configurations on the triangles are two-in one-out and one-in two-out. Here, each configuration is three-fold degenerate. A total spin state in the system is expected to have the macroscopic degeneracy since there still exist many degrees of freedom that each triangle satisfies either two-in one-out or one-in two-out. From the analogy with the spin ice systems, ${ }^{13}$ an "ice state" in the DESI system is defined as a state with the macroscopic degeneracy and the two types of spin configurations, i.e., two-in one-out and one-in two-out.

We can characterize the ice state by using a vector spin chirality $\boldsymbol{v}_{i}$ defined by

$$
\boldsymbol{v}_{i}=-\frac{2}{\sqrt{3}}\left(\boldsymbol{S}_{i_{1}} \times \boldsymbol{S}_{i_{2}}+\boldsymbol{S}_{i_{2}} \times \boldsymbol{S}_{i_{3}}+\boldsymbol{S}_{i_{3}} \times \boldsymbol{S}_{i_{1}}\right)
$$

where $i_{1}, i_{2}$ and $i_{3}$ represent three sites of the triangles in the kagomé lattice shown in Fig. 2(a). Here, we set $\left|\boldsymbol{S}_{i_{\alpha}}\right| \equiv 1(\alpha=1,2$ and 3$)$. $\boldsymbol{v}_{i}$ has only $z$-component since localized spins in the DESI system are coplanar, where we consider that the kagomé lattice is spread in $x y$-plane. $v_{i}^{z}$ has -3 (three-in and three-out) or 1 (one-in two-out and two-in one-out). It is expected that the thermal average of the vector spin chirality $\left\langle v_{i}^{z}\right\rangle$ vanishes if the four types of configurations are realized equally. On the other hand, $\left\langle v_{i}^{z}\right\rangle$ approaches unity when a large number of spin configurations for triangles are two-in one-out and one-in two-out. Furthermore, we define a fluctuation of $v_{i}^{z}$, which is given in the form

$$
\chi_{i}^{z}=\frac{\left\langle\left(v_{i}^{z}\right)^{2}\right\rangle-\left\langle v_{i}^{z}\right\rangle^{2}}{T} .
$$

To see the existence of the ice state, we calculated the total vector chirality $v_{z}=\sum_{i}\left\langle v_{i}^{z}\right\rangle / N_{\mathrm{T}}$ and the total fluctuation $\chi_{z}=\sum_{i} \chi_{i}^{z} / N_{\mathrm{T}}$. Here $N_{\mathrm{T}}$ represents the number of triangles on the 

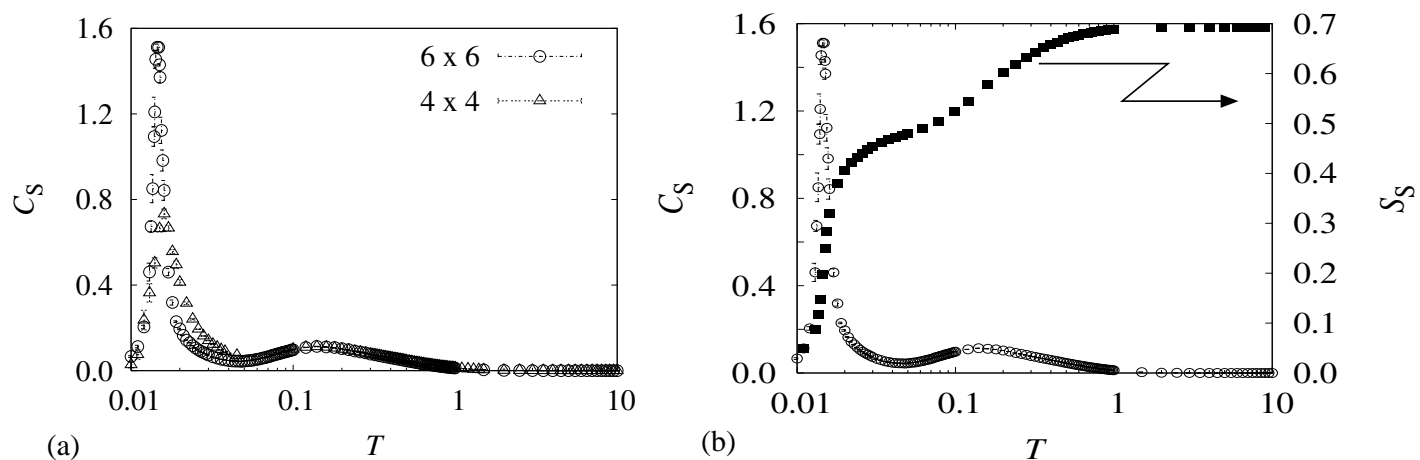

Fig. 3. (a) Temperature dependence of the specific heat, $C_{\mathrm{S}}$, for the system with $4 \times 4(=48$ sites $)$ $(\triangle)$ and $6 \times 6(=108$ sites $)(\square)$ unit cells at $\mu=0$. The specific heat has a double-peak structure, a broad peak $(T \sim 0.2)$ and a sharp peak $(T \sim 0.015)$. (b) Temperature dependence of the specific heat, $C_{\mathrm{S}}$, and the entropy, $S_{\mathrm{S}},(\mathbf{\square})$ for the system with $6 \times 6$ unit cells.

kagomé lattice which is given by $\frac{2}{3} N_{\mathrm{S}}$, where $N_{\mathrm{S}}$ is the number of sites. The summation runs over all up- and down-triangles on the kagomé lattice.

One may think that the DESI model on the kagomé lattice could be completely mapped to the n.n. AF Ising model on the same lattice, which has a macroscopically degenerate ground state. ${ }^{2,3}$ However, the interaction of the DESI system are determined by the kinetics of electrons, and it is expected that the behavior of the DESI model is different from that of the frustrated Ising spin system with the n.n. interaction.

\section{Results : Thermodynamics of the Double-Exchange Spin Ice System on the Kagomé Lattice}

In this section, we present the Monte Carlo (MC) results of the DESI model on the kagomé lattice at finite temperatures. We applied a MC technique to the calculation of thermodynamic quantities by using the grand canonical ensemble [see Appendix]. We apply the Metropolis algorithm for the updates of spin configurations and typically run 100,000 MC steps for measurement after 10,000 thermalization steps.

In ref. 11, we have already reported the dodecamer order of localized spins at sufficiently low temperatures. The dodecamer order is realized in a wide range of $\mu$, which is roughly estimated as $-0.3 \lesssim \mu \lesssim 0.2$. Thus, the DESI models around $\mu \sim 0$ are expected to show qualitatively similar behaviors. Therefore, we treat the DESI model at $\mu=0$, hereafter.

Let us investigate the behaviors on the DESI model at finite temperatures. In order to see how the entropy of localized spins is released in the system, we have investigated a specific heat and localized spin configurations. As explained in Appendix, the specific heat of the system can be decomposed into an electronic part $C_{\mathrm{E}}(T)$ and a localized spin part $C_{\mathrm{S}}(T)$. The information of localized spin configurations is included in $C_{\mathrm{S}}(T)$. In particular, we are 
interested in the behaviors of the localized spins at low temperatures. Thus, we only focus on $C_{\mathrm{S}}(T)$, which is defined as the specific heat per site hereafter. In Fig. 3(a), we show the temperature dependences of $C_{\mathrm{S}}(T)$, for the system with $4 \times 4$ unit cells $\left(N_{\mathrm{S}}=48\right)$ and $6 \times 6$ unit cells $\left(N_{\mathrm{S}}=108\right)$ at $\mu=0 . C_{\mathrm{S}}(T)$ has a double-peak structure: A broad peak $(T \sim 0.2)$ and a sharp one $(T \sim 0.015)$. In Fig. 3(b), the temperature dependence of the $C_{\mathrm{S}}$ and the entropy $S_{\mathrm{S}}$ obtained from $C_{\mathrm{S}}$ for the system with $6 \times 6$ unit cells are shown. These results provide us a picture that there exist two steps to release the entropy in the DESI system.

\subsection{Crossover to the ice state}

Let us consider the broad peak in $C_{\mathrm{S}}(T)$ at $T \sim 0.2$. As mentioned in Sec. 2.3, it is expected that the system exhibits an ice state as temperature is lowered. We speculate that the broad peak in $C_{\mathrm{S}}(T)$ is associated with the formation of the ice state. In order to clarify this idea, we investigate the entropy per site $S_{\mathrm{S}}(T)$ from $C_{\mathrm{S}}(T)$,

$$
S_{\mathrm{S}}(T)=\log 2-\int_{T}^{\infty} \frac{C_{\mathrm{S}}\left(T^{\prime}\right)}{T^{\prime}} d T^{\prime} .
$$

Here we use $S_{\mathrm{S}}(\infty)=\log 2$, since all spin configurations with the number of states $2^{N_{\mathrm{S}}}$ can be realized in the high temperature limit $(T=\infty)$. The data for $S_{\mathrm{S}}(T)$ are shown in Fig. 3(b).

In the high temperature region, $S_{\mathrm{S}}(T)$ deviates from $\log 2$ at $T \lesssim 1$, since the interactions are mediated by the DE mechanism. In the intermediate temperature region, we see that $S_{\mathrm{S}}(T)$ has a plateau structure at $T \sim 0.05$ with $S_{\mathrm{S}}(T) \simeq 0.5$. Since the spin ice system on a kagomé lattice can be mapped to an AF Ising model on the identical lattice, the entropy of the ice state for the DESI system is roughly estimated from the residual entropy of the AF

Ising model on the kagomé lattice, ${ }^{3} S_{\text {Ising }} \simeq 0.5$, which is consistent with the observed value for $S_{\mathrm{S}}$.

An alternative clarification is given by the temperature dependence of $v_{z}$ and $\chi_{z}$. The results for the system with $6 \times 6$ unit cells (108 sites) and $\mu=0$ are shown in Fig. $4 . \chi_{z}$ has the maximum value at $T \sim 0.2$, and then, below which it rapidly decreases until $T \sim 0.05$. Similarly, it is found that $v_{z}$ has a inflection point at around $T \sim 0.2$, and saturates below $T \sim 0.05$.

These results suggest that the broad peak in $C_{\mathrm{S}}(T)$ at $T \sim 0.2$ is due to a crossover to the ice state. At $T \sim 0.05$, the ice state is stabilized, where a macroscopic number of thermally degenerate states still remain. From the analogy with the spin ice system, ${ }^{13}$ it is considered that the origin of the ice state in the DESI system is the short-range (n.n.) interaction between localized spins. The ice state in which a large number of the entropy remains is indicated by the broad peak in the specific heat and is driven by the short-range ferromagnetic interaction.

\subsection{Transition to the dodecamer ordered state}

In this section, we focus on the sharp peak in the specific heat. The sharp peak means further entropy release in the system which results from the reduction of degrees of freedom 


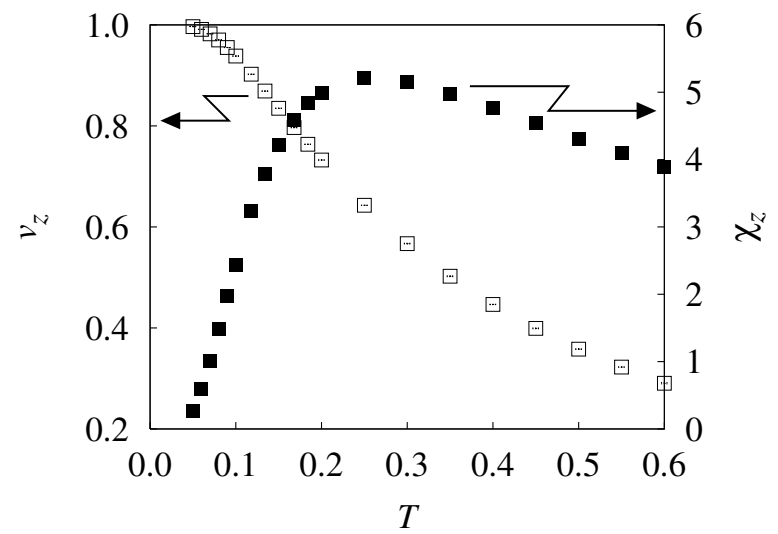

Fig. 4. Temperature dependence of the vector spin chirality $v_{z}(\square)$ and the susceptibility of the chirality $\chi_{z}(\mathbf{\square})$ for the system with $6 \times 6$ unit cells at $\mu=0 . v_{z}$ approaches unity as the temperature is lowered. $\chi_{z}$ has a broad peak at $T \sim 0.25$.

for localized spins. As an origin of such an entropy release, it is considered that some kind of ordering of spins are generated due to the residual effects in addition to the short-range interaction in the model. An effective long-range interaction due to the kinetic energy gain of electrons is one candidate for such effects, since higher order hopping process of electrons is considered to be important at low temperatures. Indeed, the dodecamer order driven by the kinetic energy gain of electrons appears at low temperatures in the system. ${ }^{11}$ Thus, it is expected that the sharp peak corresponds to a change from the ice state to the dodecamer ordered state.

The order parameter for the dodecamer formation is characterized by using bond correlations, and is defined by

$$
d_{i} \equiv \frac{1}{18}\left(-\sum_{n=1}^{12} \tau_{i_{n}} \tau_{i_{n+1}}+\sum_{m=1}^{6} \tau_{i_{2 m}} \tau_{i_{2 m+2}}\right),
$$

where a site $i_{n}$ is indicated in Fig. 1(b) and we set $i_{13} \equiv i_{1}, i_{14} \equiv i_{2}$. Note that the order parameter $d_{i}$ is a unity when the dodecamer is formed. The dodecamer structure factor is given in a form

$$
D_{\boldsymbol{q}}=\frac{1}{N_{\mathrm{C}}} \sum_{i, j} e^{i \boldsymbol{q} \cdot\left(\boldsymbol{r}_{i}-\boldsymbol{r}_{j}\right)}\left\langle d_{i} d_{j}\right\rangle,
$$

where $N_{\mathrm{C}}$ is the number of unit cells $\left(N_{\mathrm{C}} \equiv \frac{1}{3} N_{\mathrm{S}}\right)$ and $\sum_{i, j}$ represents the summation for any pairs of the order parameter shown in Fig. 1(a). $D_{\boldsymbol{q}}$ has three independent maximum peaks at $\boldsymbol{Q}_{1}={ }^{t}(0,2 \pi / \sqrt{3} a), \boldsymbol{Q}_{2}={ }^{t}(\pi / a, \pi / \sqrt{3} a)$ and $\boldsymbol{Q}_{3}={ }^{t}(\pi / a,-\pi / \sqrt{3} a)$, those characterize the dodecamer order. An average of maximum peaks, $D_{Q} \equiv\left\{D_{Q_{1}}+D_{Q_{2}}+D_{Q_{3}}\right\} / 3$, diverges increasing the system size at sufficiently low temperatures. ${ }^{11}$ This result indicates that the dodecamer order is realized in the DESI system on the kagomé lattice. Note that the change 


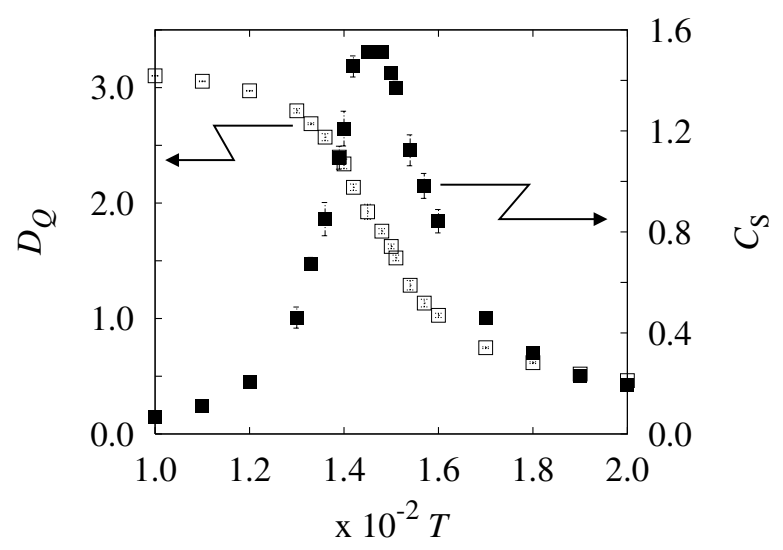

Fig. 5. Temperature dependence of $D_{Q}$ and specific heat $C_{\mathrm{S}}$ for the system with $6 \times 6$ unit cells and $\mu=0$.

from the ice state to the dodecamer ordered state is a phase transition since the translational symmetry is broken due to the dodecamer ordering.

Temperature dependences of $D_{Q}$ and specific heat $C_{\mathrm{S}}$ for $N_{\mathrm{C}}=6 \times 6$ and $\mu=0$ are also shown in Fig. 5. $D_{Q}$ increases rapidly at around the sharp peak temperature in the specific heat. The temperature at the sharp peak is almost consistent with the inflection point of $D_{Q}$. Thus, we conclude that the sharp peak corresponds to a transition to the dodecamer order driven by the effective long-range interaction due to the kinetic energy gain. In fact, the majority of the entropy release in the DESI system is caused by the dodecamer order as shown in Fig. 3(b). The similar behavior is observed in the system with $4 \times 4$ unit cells. Note that the sharp peak is smeared out by the finite-size effect.

\subsection{Stability of the dodecamer ordered state}

A stability and an origin for the dodecamer ordered state have been discussed in Ref. 11. Let us now present brief summary. (1) The dodecamer order is realized in a wide doping region $1 / 3 \lesssim n \lesssim 1 / 2(-0.3 \lesssim \mu \lesssim 0.2)$, where $n$ is the number of particles per site. (2) The dodecamer order is consequence of a selection of a certain path, along which electrons can move easily, i.e., the kinetic energy gain. The result of (2) is concluded by the density of states: An energy gap is not opened by the dodecamer ordering. From this result, we concluded that the dodecamer ordered state is metallic. The result of (1) also means that the dodecamer order is not sensitive to the Fermi surface. In this way, the results of (1) and (2) are consistent with each other. Considering the DE mechanism which gains the kinetic energy by broadening the bandwidth, the weak $n$-dependence of the dodecamer ordered state is understood as follows. The ferromagnetic interaction is dominant among the localized spins at around $\mu=0$ where the kinetic energy is mostly gained. Although there is the spin structure that is characterized by the finite wave vectors, the spin structure is not originated from the nesting of the Fermi 


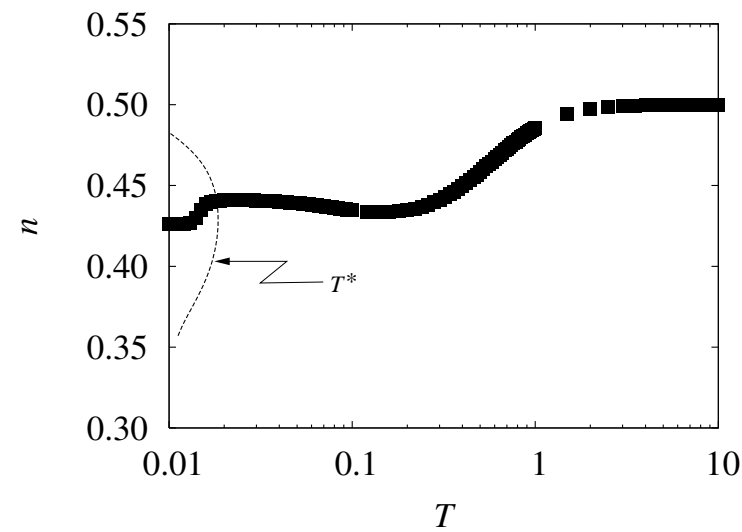

Fig. 6. Temperature dependence of $n$ for the system with $N_{\mathrm{C}}=6 \times 6$ and $\mu=0$, where black squares represent $n$ at each temperature. The dashed line represents the approximate phase boundary for the dodecamer ordered state $T^{*}$ estimated from $D_{Q}$ [see Ref. 11 for further details]. There is the two-step structure which corresponds to the crossover to the ice state and the transition to the dodecamer ordered state as well as the entropy.

surface, but from the frustration, i.e., the uniaxial anisotropy.

We show the temperature dependence of $n$ for the system with $N_{\mathrm{C}}=6 \times 6$ and $\mu=0$ in Fig. 6. The two-step behavior can be found, which corresponds to the crossover to the ice state and the phase transition to the dodecamer ordered state. $n$ decreases gradually from $T \sim 1$ to 0.2 . In the ice state, $n$ keeps the almost same values which can be regarded as a plateau structure. Going down to further low temperatures, $n$ shows a rapid reduction at around the sharp peak of $C_{\mathrm{S}}$. The behavior of $n$ is consistent with that of the entropy. The values of $n$ at $\mu=0$ are also included in the region $\frac{1}{3} \lesssim n \lesssim \frac{1}{2}$. It is expected that the temperature dependence of $n$ is not affected drastically by changing $\mu$ around zero. Thus, similar arguments are considered to be useful in the region of $\mu$ where the dodecamer ordered state is observed.

\subsection{Possibility of further transition in the dodecamer ordered phase}

In the dodecamer phase, e.g., Fig. 1(a), we can find that dodecamers are two-fold degenerate. One has out-spins on the tips, and the other has in-spins as in Fig. 7(a). Two dodecamers can be regarded as "pseudo-up" and "pseudo-down", respectively. Then the dodecamer order can be described using pseudospins on a triangular lattice. Due to the DE mechanism, a pseudo-up and a pseudo-down are connected by the two bonds with the transfer integral $t_{1}$ [see Fig. 7(b)(right)], while the same pseudospins are connected by the two bonds with the transfer integral $t_{2}$ [see Fig. 7(b)(left)]. There is an AF interaction between the pseudospins since $t_{1}>t_{2}$. Thus, in the dodecamer phase, the problem of the DESI system on the kagomé lattice can be mapped onto that of psuedospin systems with the effective AF interaction on the triangular lattice. When the random pseudospin configurations are realized, it is expected that the entropy $S_{\mathrm{S}}(T)$ is $\frac{1}{12} \log 2 \simeq 0.06$ since the dodecamer has two degrees of freedom. 

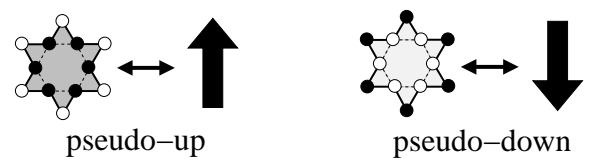

(a) two species of dodecamer and pseudospins
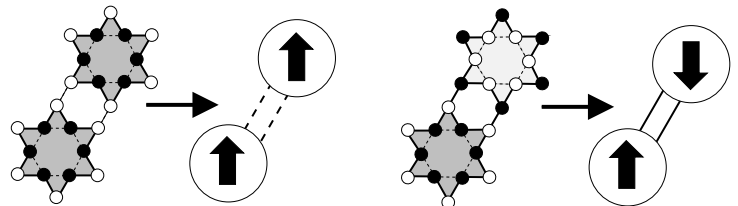

(b) inter-dodecamer interaction and pseudo-AF interaction

Fig. 7. (a) Two species of dodecamer: pseudo-up and pseudo-down. A pseudo-up and a pseudo-down have out-spins and in-spins on pointed tips of the dodecamer, respectively. (b) Inter-dodecamer interaction: An inter-dodecamer interaction behaves like the AF interaction, because there are two bonds with the transfer integral $t_{1}$ between a pseudo-up and a pseudo-down. On the other hand, there are two bonds with $t_{2}$ between the same pseudo-spins. Solid and dashed double lines represent pseudo-AF and pseudo-ferromagnetic bond, respectively.

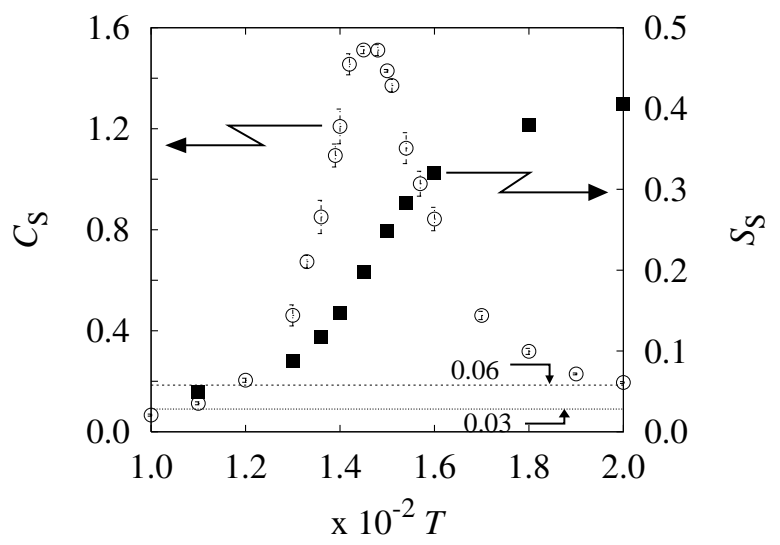

Fig. 8. Temperature dependences of the specific heat $C_{\mathrm{S}}$ and the entropy $S_{\mathrm{S}}$ of localized spins for the system with $6 \times 6$ unit cells. The upper and the lower dashed line represent $\frac{1}{12} \log 2 \simeq 0.06$ and $0.34 / 12 \simeq 0.03$, respectively, where 0.34 is the value of the residual entropy of the AF Ising model on the triangular lattice.

However, it is considered that some of the entropy $S_{\mathrm{S}}(T)$ per site are released due to the AF interaction. For example, in the AF Ising model on the triangular lattice, the exact residual entropy is known to be about 0.34 per site, ${ }^{1}$ which corresponds to the residual entropy of $0.34 / 12 \simeq 0.03$ per site for the present model. Magnified views of $C_{\mathrm{S}}$ and $S_{\mathrm{S}}$ at the sharp peak are shown in Fig. 8. From the results as in Fig. 8, it seems that $0.03 \lesssim S_{\mathrm{S}} \lesssim 0.06$ in the dodecamer phase. Thus, we may read off that there still remain degrees of freedom for local- 


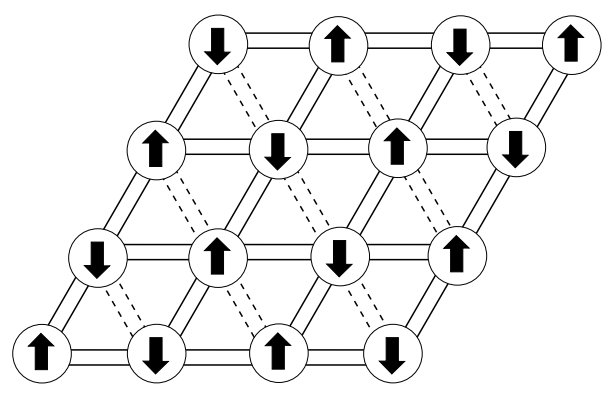

Fig. 9. The pseudo-AF phase. The uparrows and the downarrows represent the dodecamer with the out-spins on the tips and that with the in-spins, respectively.

ized spins even in the dodecamer phase. From this result, disordered psuedospin states which satisfy the AF interaction seem to appear in the dodecamer phase. However, at further low temperatures, a pseudospin ordering may be expected to be realized considering the effective long-range nature due to the $\mathrm{DE}$ mechanism. A dodecamer phase without/with a long-range order of the pseudospins is called "pseudo-para phase" / "pseudo-AF phase", hereafter. The former case has only a translational symmetry breaking due to the dodecamer order, while the latter case also has a spin symmetry breaking due to some kind of pseudospin ordering in addition to the translational symmetry breaking. In Fig. 9, we show a possible pseudo-AF phase as a candidate for the ground state. In the pseudo-AF phase, the strong bonds with the transfer integral $t_{1}$ linearly align in two directions, while the weak bonds with $t_{2}$ align in the other direction. The pseudo-AF phase has a rotational symmetry breaking in the lattice space in addition to the translational and the spin symmetry breaking. In the pseudo-AF phase, further kinetic energy gain may be expected because of the linear alignment of the strong bonds in two direction, that is, the motion of electrons on the pseudo-square lattice will be possible. As far as the DESI system with $4 \times 4$ and $8 \times 8$ unit cells has been investigated, our numerical calculations indicate that the pseudo-AF phase has the smallest energy in all dodecamer ordered states, i.e., the ground state of the DESI model on the kagomé lattice. According to these considerations, we expect that there is a possibility of a phase transition in the dodecamer ordered state, i.e., a transition from the pseudo-para phase to the pseudo-AF phase. However, the ground state in the thermodynamic limit cannot be clarified so far, and more detailed analysis at low temperatures for larger system sizes are needed.

\section{Discussion and Summary}

In the DESI system on the kagomé lattice, the long-range interaction due to the DE mechanism plays an important role to stabilize the dodecamer ordered state. In fact, a similar situation has been seen in the spin ice system. ${ }^{13}$ The spin ice system has been discussed by two models: One has a ferromagnetic interaction due to the n.n. Ising interaction, and the other has that due to the long-range dipolar interaction. In particular, the latter model is called a 


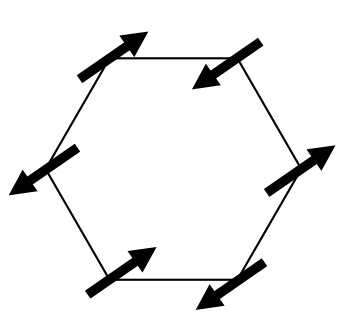

(a) hexamer

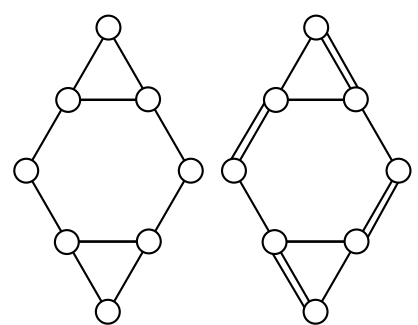

(b) octamer

Fig. 10. (a) Hexamer in $\mathrm{ZnCr}_{2} \mathrm{O}_{4}$. The $S=3 / 2$ localized spins align antiferromagnetically in an arbitrary direction. (b) Octamers in $\mathrm{CuIr}_{2} \mathrm{O}_{4}$ : (left) Octamer composed of eight $\operatorname{Ir}^{3+}(S=0)$ which is represented by open circles. (right) Octamer composed of eight $\operatorname{Ir}^{4+}(S=1 / 2)$ which is represented by open circles, where the double lines represent the dimer-singlets of $\operatorname{Ir}^{4+}$.

dipolar spin ice model. According to MC calculations in the dipolar spin ice model, a doublepeak structure in a specific heat has been observed as in the DESI system: A broad peak at higher temperature and a sharp peak at lower temperature. ${ }^{15}$ The sharp peak corresponds to the spin cluster ordered state. Such a cluster ordered state is not observed in the spin ice model with the n.n. Ising interaction. These results indicate the long-range nature of the interaction plays an important role to create a cluster ordering even in the frustrated spin systems as well as the electron systems. Although the spin ice system is an insulator, the DESI system is considered to be metallic. In this way, a cluster ordering might be generic to frustrated systems. In general, the frustration suppresses the usual ordered states and leads to the degenerate states at low temperatures. In the frustrated systems, the residual interaction selects the cluster ordered state among the macroscopic degenerate states.

Let us consider a possibility of the cluster order in realistic systems with the frustration. As mentioned in the introduction, there are several examples of a cluster order in nature: the hexamer in $\mathrm{ZnCr}_{2} \mathrm{O}_{4}{ }^{9}$ [see Fig. 10(a)], the octamer in $\mathrm{CuIr}_{2} \mathrm{~S}_{4}{ }^{10}$ [see Fig. 10(b)] and so forth. In the cubic spinel $\mathrm{ZnCr}_{2} \mathrm{O}_{4}, \mathrm{Cr}^{3+}(S=3 / 2)$ resides on the pyrochlore structure and interact with each other antiferromagnetically. ${ }^{16}$ At low temperatures, a hexagonal AF spin cluster formation is realized and the pyrochlore sites are tiled by the hexamers. ${ }^{9}$ In a thiospinel $\mathrm{CuIr}_{2} \mathrm{~S}_{4}$, mixed-valence states of $\operatorname{Ir}^{3+}(S=0)$ and $\operatorname{Ir}^{4+}(S=1 / 2)$ are realized in the insulating phase at low temperatures and they occupy octahedral sites. ${ }^{17-19}$ In this case, the system have a charge frustration as well as a spin frustration. In the insulating phase, Ir sites are tiled by two kinds of octamers: One consists of eight $\mathrm{Ir}^{3+}$ and the other consists of eight $\mathrm{Ir}^{4+}$ with four dimer-singlets. ${ }^{10}$ In this way, the cluster order can be realized in realistic frustrated systems. Such a cluster order may be explained by including the effect of the residual interactions which are neglected in the non-frustrated system.

In conclusion, there are at least two steps for the entropy release in the DESI system on the kagomé lattice, which is indicated by the peak structures in the specific heat. The ice state 
appears at intermediate temperatures due to the n.n. ferromagnetic interaction. From the estimated entropy, it has been clarified that there is a large number of thermally degenerate states in the ice state. As the temperature is lowered, a large part of the entropy in the system is released by the dodecamer ordering with the translational symmetry breaking. The dodecamer order is driven by the kinetic energy gain and the frustration: The dodecamer is the kinetic-energetically stable path for electrons. Furthermore, a possibility of a transition in the dodecamer phase is indicated by our MC results: (1) The estimated entropy $S_{\mathrm{S}}$ seems to remain in the dodecamer ordered phase because of the existence of two kinds of dodecamers which can be regarded as the pseudospins. (2) The pseudo-AF phase is the ground state in the system with small-size clusters. These result possibly indicate that there is a transition from the pseudo-para phase to the pseudo-AF phase to gain the further kinetic energy. It is expected that the entropy in the system will be completely released by the ordering of the pseudospins, i.e., two kinds of dodecamers. However, more detailed analysis are needed to clarify this point.

\section{Acknowledgements}

We acknowledge M. Miyazaki for sincerely reading of our paper. We would like to thank S. Katsurada and Y. Shinohara for computational supports. The numerical computations have been performed mainly using the facilities of the super AOYAMA+ project (SAPP). This work was partially supported by a Grant-in-Aid for 21st COE program from the Ministry of Education, Culture, Sports, Science and Technology of Japan.

\section{Appendix: Monte Carlo Method}

Here, we explain the application of the MC method for the Hamiltonian (5) in detail. ${ }^{20}$ We define abbreviations $\left\{c_{i}, c_{i}^{\dagger}\right\} \equiv\left(c_{1}, \cdots, c_{N_{\mathrm{S}}}, c_{1}^{\dagger}, \cdots, c_{N_{\mathrm{S}}}^{\dagger}\right)$ and $\left\{\tau_{i}\right\} \equiv\left(\tau_{1}, \cdots, \tau_{N_{\mathrm{S}}}\right)$ which represent degrees of freedom for electrons and localized spins (adiabatic fields), respectively. Using Eq. (5), the partition function of the system in the grand canonical ensemble is written as

$$
\begin{aligned}
Z & =\operatorname{Tr}_{\mathrm{C}} \operatorname{Tr}_{\mathrm{F}} \exp \left[-\beta\left\{\sum_{\langle i, j\rangle} t\left(\tau_{i}, \tau_{j}\right)\left(c_{i}^{\dagger} c_{j}+\text { h.c. }\right)-\mu \sum_{i} c_{i}^{\dagger} c_{i}\right\}\right], \\
& =\operatorname{Tr}_{\mathrm{C}} \operatorname{Tr}_{\mathrm{F}} \exp \left[-\beta\left(\hat{H}_{\mathrm{K}}-\mu \sum_{i} c_{i}^{\dagger} c_{i}\right)\right],
\end{aligned}
$$

where $\operatorname{Tr}_{\mathrm{F}}$ and $\operatorname{Tr}_{\mathrm{C}}$ represent traces over $\left\{c_{i}, c_{i}^{\dagger}\right\}$ and $\left\{\tau_{i}\right\}$, respectively. And, $\mu$ represents the chemical potential. Here, we define the kinetic energy part of the Hamiltonian as $\hat{H}_{\mathrm{K}} \equiv$ $\sum_{\langle i, j\rangle} t\left(\tau_{i}, \tau_{j}\right)\left(c_{i}^{\dagger} c_{j}+\right.$ h.c. $)$. Once a localized spin configuration $\left\{\tau_{i}\right\}$ is given, it is easy to diagonalize the Hamiltonian (5) because it is represented by the quadratic form of fermion creation and annihilation operators, i.e., $N_{\mathrm{S}} \times N_{\mathrm{S}}$ matrix: $\hat{H}_{\mathrm{K}}=\sum_{\langle i, j\rangle} c_{i}^{\dagger} H_{i j} c_{j}$, where $H_{i j} \equiv$ $t\left(\tau_{i}, \tau_{j}\right)$ for n.n. pairs of $i$ and $j$, otherwise 0 . Thus, the trace operation $\operatorname{Tr}_{\mathrm{F}}$ is easily performed 
J. Phys. Soc. Jpn.

Full PaPer

through the energy eigenvalues $\varepsilon_{k}\left(\left\{\tau_{i}\right\}\right)$ of $\hat{H}_{\mathrm{K}}$ for a given configuration $\left\{\tau_{i}\right\}$. Finally, the partition function $(\mathrm{A} \cdot 2)$ can be written as

$$
Z=\operatorname{Tr}_{\mathrm{C}} \exp \left[-\beta F_{\mathrm{eff}}\left(\left\{\tau_{i}\right\}\right)\right]
$$

with the effective free energy

$$
F_{\text {eff }}\left(\left\{\tau_{i}\right\}\right)=-k_{\mathrm{B}} T \sum_{k=1}^{N_{\mathrm{S}}} \log \left(1+\exp \left\{-\beta\left[\varepsilon_{k}\left(\left\{\tau_{i}\right\}\right)-\mu\right]\right\}\right) .
$$

Probability distribution of a certain configuration $\left\{\tau_{i}\right\}$ is given by

$$
P\left(\left\{\tau_{i}\right\}\right)=\exp \left[-\beta F_{\text {eff }}\left(\left\{\tau_{i}\right\}\right)\right] / Z,
$$

whose numerator is regarded as the Boltzmann factor.

Thermal average of arbitrary observable $\hat{A}\left(\left\{c_{i}, c_{i}^{\dagger}\right\},\left\{\tau_{i}\right\}\right)$ is given as follows in the grand canonical ensemble:

$$
\left\langle\hat{A}\left(\left\{c_{i}, c_{i}^{\dagger}\right\},\left\{\tau_{i}\right\}\right)\right\rangle \equiv \frac{\operatorname{Tr}_{\mathrm{C}} \operatorname{Tr}_{\mathrm{F}}\left[\hat{A}\left(\left\{c_{i}, c_{i}^{\dagger}\right\},\left\{\tau_{i}\right\}\right) \mathrm{e}^{-\beta \hat{H}}\right]}{\operatorname{Tr}_{\mathrm{C}} \operatorname{Tr}_{\mathrm{F}}\left[\mathrm{e}^{-\beta \hat{H}}\right]} .
$$

Using Eq. (A.5), the thermal average is also written as

$$
\left\langle\hat{A}\left(\left\{c_{i}, c_{i}^{\dagger}\right\},\left\{\tau_{i}\right\}\right)\right\rangle=\left\langle\left\langle\hat{A}\left(\left\{c_{i}, c_{i}^{\dagger}\right\},\left\{\tau_{i}\right\}\right)\right\rangle_{\mathrm{F}}\right\rangle_{\mathrm{C}},
$$

where $\langle\cdots\rangle_{\text {F }}$ represents the grand canonical average for a fixed configuration $\left\{\tau_{i}\right\}$ defined by

$$
\langle\cdots\rangle_{\mathrm{F}} \equiv \frac{\operatorname{Tr}_{\mathrm{F}}\left[\cdots \mathrm{e}^{-\beta \hat{H}}\right]}{\operatorname{Tr}_{\mathrm{F}}\left[\mathrm{e}^{-\beta \hat{H}}\right]}
$$

while $\langle\cdots\rangle_{\mathrm{C}}$ is the thermal average over configurations of $\left\{\tau_{i}\right\}$ defined by

$$
\langle\cdots\rangle_{\mathrm{C}} \equiv \operatorname{Tr}_{\mathrm{C}}\left[\cdots P\left(\left\{\tau_{i}\right\}\right)\right] .
$$

Note that, in the thermal average for observables without the electron operator $\left\{c_{i}, c_{i}^{\dagger}\right\}$, e.g., $v_{i}^{z}$ (Eq. (7)) and $d_{i}$ (Eq. (10)), $\langle\cdots\rangle$ becomes $\langle\cdots\rangle_{\mathrm{C}}$.

The specific heat is defined as a fluctuation of the energy in the form

$$
C(T)=\frac{\left\langle\hat{H}^{2}\right\rangle-\langle\hat{H}\rangle^{2}}{T^{2}} .
$$

$C(T)$ can be decomposed into electronic part $C_{\mathrm{E}}(T)$ and spin part $C_{\mathrm{S}}(T)$ as follows:

$$
C(T)=C_{\mathrm{E}}(T)+C_{\mathrm{S}}(T)
$$

where

$$
\begin{gathered}
C_{\mathrm{E}}(T) \equiv \frac{1}{T^{2}}\left\langle E_{2}-E_{1}^{2}\right\rangle_{\mathrm{C}}, \\
C_{\mathrm{S}}(T) \equiv \frac{1}{T^{2}}\left(\left\langle E_{1}^{2}\right\rangle_{\mathrm{C}}-\left\langle E_{1}\right\rangle_{\mathrm{C}}^{2}\right) .
\end{gathered}
$$




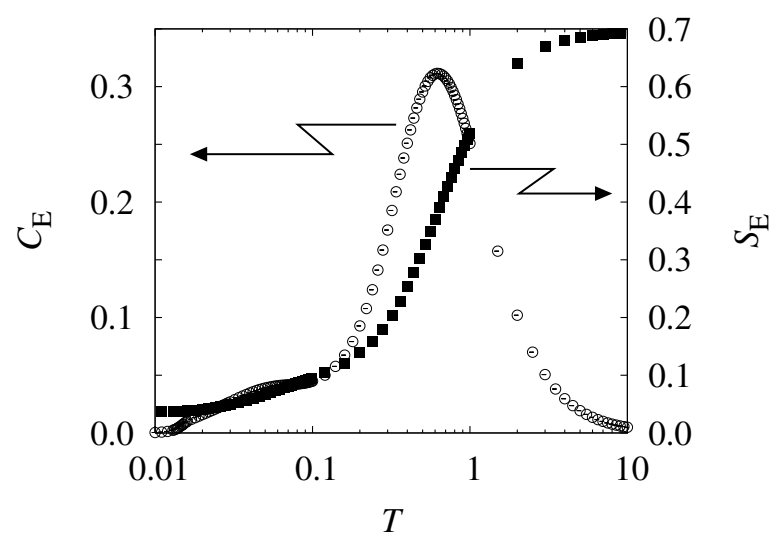

Fig. A.1. Temperature dependence of $C_{\mathrm{E}}$ and $S_{\mathrm{E}} \cdot C_{E}$ has a peak at $T \simeq 0.6$, which is almost the same order of $t_{1}$ and $t_{2}$.

Here, we set $E_{1} \equiv\langle\hat{H}\rangle_{\mathrm{F}}$ and $E_{2} \equiv\left\langle\hat{H}^{2}\right\rangle_{\mathrm{F}}$. Eq. (A.12) is given by the electronic energy fluctuation averaged over configurations $\left\{\tau_{i}\right\}$, while Eq. (A.13) is given by the energy fluctuations among spin configurations $\left\{\tau_{i}\right\}$. Thus, it is easily found that Eq. (A.12) does not include fluctuation of localized spins while Eq. (A.13) does not include fluctuation of electrons. $E_{1}$ and $E_{2}$ are calculated as

$$
\begin{aligned}
E_{1} & =\sum_{k} \varepsilon_{k}\left(\left\{\tau_{i}\right\}\right) f_{\mathrm{F}}\left(\varepsilon_{k}\left(\left\{\tau_{i}\right\}\right)\right) \\
E_{2} & =\sum_{k} \varepsilon_{k}\left(\left\{\tau_{i}\right\}\right)^{2} f_{\mathrm{F}}\left(\varepsilon_{k}\left(\left\{\tau_{i}\right\}\right)\right) \\
& +\sum_{k \neq l} \varepsilon_{k}\left(\left\{\tau_{i}\right\}\right) \varepsilon_{l}\left(\left\{\tau_{i}\right\}\right) f_{\mathrm{F}}\left(\varepsilon_{k}\left(\left\{\tau_{i}\right\}\right)\right) f_{\mathrm{F}}\left(\varepsilon_{l}\left(\left\{\tau_{i}\right\}\right)\right) .
\end{aligned}
$$

where $f_{\mathrm{F}}(x)$ is the Fermi distribution function given in the form,

$$
f_{\mathrm{F}}(x)=\frac{1}{\mathrm{e}^{\beta(x-\mu)}+1} .
$$

In the grand canonical ensemble, the entropy of electronic part per site in the DESI system, $S_{\mathrm{E}}(T=\infty)$ is $\log 2$ as follows:

$$
\begin{aligned}
S_{\mathrm{E}}(T=\infty) & =\int_{0}^{\infty} d T \frac{C_{\mathrm{E}}(T)}{T} \\
& =\left\langle\int_{0}^{\infty} d T \frac{1}{T^{3}}\left(E_{2}-E_{1}^{2}\right)\right\rangle_{\mathrm{C}} \\
& =\langle\log 2\rangle_{\mathrm{C}} \\
& =\log 2 .
\end{aligned}
$$

In the DESI system, the entropy of localized spin is $\log 2$, since each localized spin has two degrees of freedom. Therefore, total entropy per site in the DESI system is $\log 4$. Temperature dependences of $C_{\mathrm{E}}$ and $S_{\mathrm{E}}$ for the system with $6 \times 6$ unit cells are shown in Fig. A.1. A peak 
can be found at around $T=t_{1} \sim t_{2}$ in Fig. A.1. In the electronic part of the specific heat, $C_{\mathrm{E}}$, any remarkable structures are not observed at sufficiently low temperatures $T \sim 0.01$ where the dodecamer order is realized. 


\section{References}

1) G. H. Wannier: Phys. Rev. 79 (1950) 357.

2) I. Syozi: Prog. Theor. Phys. 6 (1951) 306.

3) K. Kano and S. Naya: prog. Theor. Phys. 10 (1953) 158.

4) P. W. Anderson: Phys. Rev. 102 (1956) 1008.

5) C. K. Majumdar and D. K. Ghosh: J. Math. Phys. 10 (1969) 1399.

6) B. S. Shastry and B. Sutherland: Physica B 108 (1981) 1069.

7) H. Kageyama, K. Yoshimura, R. Stern, N. Mushnikov, M. Kato, K. Kosuge, C. Slichter, T. Goto and Y. Ueda: Phys. Rev. Lett. 82 (1999) 3168.

8) S. Miyahara and K. Ueda: Phys. Rev. Lett 82 (1999) 3701.

9) S.-H. Lee, C. Broholm, W. Ratcliff, G. Gasparovic, Q. Huang, T. H. Kim and S.-W. Cheong: Nature 418 (2002) 856.

10) P. G. Radaelli, Y. Horibe, M. J. Gutmann, H. Ishibashi, C. H. Chen, R. M. Ibberson, Y. Koyama, Y. S. Hor, V. Kiryukhin and S.-W. Cheong: Nature 416 (2002) 155.

11) Y. Shimomura, S. Miyahara and N. Furukawa: J. Phys. Soc. Jpn. 73 (2004) 1623.

12) C. Zener: Phys. Rev. 82 (1951) 403.

13) S. T. Bramwell and M. J. P. Gingras: Science 294 (2001) 1495, and references therein.

14) P. W. Anderson and H. Hasegawa: Phys. Rev. 100 (1955) 675.

15) R. G. Melko, B. C. den Hertog and M. J. P. Gingras: Phys. Rev. Lett. 87 (2001) 67203.

16) S.-H. Lee, C. Broholm, T. H. Kim, W. Ratcliff, and S.-W. Cheong: Phys. Rev. Lett. 84 (2000) 3718 .

17) T. Oda, M. Shirai, N. Suzuki and K. Motizuki: J. Phys. Condens. Matter. 7 (1995) 4433.

18) K. Kumagai, S. Tsuji, T. Hagino and S. Nagata: Spectroscopy of Mott insulators and Correlated Materials, edited by A. Fujimori and Y. Tokura (Springer, Berlin, 1995).

19) J. Matsuno, T. Mizokawa, A. Fujimori, D. A. Zatsepin, V. R. Galakhov, E. Z. Kurmaev, Y. Kato and S. Nagata: Phys. Rev. B 55 (1997) R15 979

20) S. Yunoki, J. Hu, A. L. Malvezzi, A. Moreo, N. Furukawa and E. Dagotto: Phys. Rev. Lett. 80 (1998) 845. 